\title{
Combining Tissue and Circulating Tumor DNA Increases the Detection Rate of CTNNB1 Mutation in Hepatocellular Carcinoma
}

Stine Karlsen Oversoe ( $\sim$ stinkarl@rm.dk)

Aarhus Universitetshospital https://orcid.org/0000-0001-9762-1679

Michelle Simone Clement

Department of Clinical Biochemistry, Aarhus University Hospital

\section{Britta Weber}

Department of Clinical Oncology and Danish Centre of Particle Therapy, Aarhus University Hospital

Henning Grønbæk

Department of Hepatology and Gastroenterology, Aarhus University Hospital

Stephen Jacques Hamilton-Dutoit

Department of Pathology, Aarhus University Hospital

\section{Boe Sandahl Sorensen}

Department of Clinical Biochemistry, Aarhus University Hospital

\section{Jens Kelsen}

Department of Hepatology and Gastroenterology, Aarhus University Hospital

\section{Research article}

Keywords: hepatocellular carcinoma, molecular pathology, circulating tumor DNA, droplet digital PCR, predictive biomarkers

Posted Date: October 9th, 2020

DOI: https://doi.org/10.21203/rs.3.rs-88929/v1

License: (c) (i) This work is licensed under a Creative Commons Attribution 4.0 International License. Read Full License

Version of Record: A version of this preprint was published at BMC Cancer on April 8th, 2021. See the published version at https://doi.org/10.1186/s12885-021-08103-0. 


\section{Abstract}

Background and aims: Studies suggest that mutations in the CTNNB1 gene are predictive of response to immunotherapy, an emerging therapy for advanced hepatocellular carcinoma (HCC). Analysis of circulating tumor DNA (ctDNA) offers the possibility of serial non-invasive mutational profiling of tumors. Combining tumor tissue and ctDNA analysis may increase the detection rate of mutations.

This study aimed to evaluate the frequency of the CTNNB1 p.T41A mutation in ctDNA and tumor samples from HCC patients and to evaluate the concordance rates between plasma and tissue. We further evaluated changes in ctDNA after various HCC treatment modalities and the impact of the CTNNB1 p.T41A mutation on the clinical course of HCC.

Methods: We used droplet digital PCR to analyze plasma from 95 patients and the corresponding tumor samples from 37 patients during three years of follow up.

Results: In tumor tissue samples, the mutation rate was 8.1\% (3/37). In ctDNA from HCC patients, we found a CTNNB1 mutation rate of 9.5\% (9/95) in the pre-treatment samples. Adding results from plasma analysis to the subgroup of patients with available tissue samples, the mutation detection rate increased to $13.5 \%(5 / 37)$. There was no difference in overall survival according to CTNNB1 mutational status. Serial testing of ctDNA indicated a clonal evolution of $\mathrm{HCC}$ or arising of multicentric tumors with separate genetic profiles.

Conclusion: Combining analysis of ctDNA and tumor tissue increased the detection rate of CTNNB1 mutation in HCC patients. A liquid biopsy approach may be useful in a tailored therapy of HCC.

\section{Introduction}

Hepatocellular carcinoma (HCC) is a severe disease with significant morbidity and high mortality[1]. Treatment of HCC is guided by tumor stage, underlying liver disease and performance status. For the past decade, systemic treatment options were limited to sorafenib but recently, other tyrosine-kinase inhibitors demonstrated a survival benefit in advanced $\mathrm{HCC}[2,3]$. In addition, new treatment strategies with immunotherapy for advanced HCC are emerging[4]. As many treatments will be available for patients with advanced HCC, clinicians need methods to select the right treatment for the right patient.

Pronounced intratumor heterogeneity and the presence of multicentric tumors challenge a global tumor molecular profiling from a single biopsy[5, 6]. In addition, mutational profiling of tumor biopsies taken at an early stage of HCC do not necessarily provide optimal guidance for treatment decisions later in the disease course. Conversely, ctDNA potentially represents the whole tumor burden and may provide information on specific tumor mutations during a clonal evolution. ctDNA analysis is non-invasive and can be performed repeatedly during the course of disease and thereby provide an accurate picture of the tumor mutation profile before clinical decision-making. 
CTNNB1 mutations belong to the most prevalent genetic alterations in HCC[7]. The p.T41A mutation is detected in $10-15 \%$ of tissue samples from primary tumors and leads to activation of the $\mathrm{Wnt} / \beta$-catenin signaling pathway[8]. CTNNB1 mutations can appear at any time in tumor evolution[9], and Vilarinho et al. suggested a progressive clonal evolution in the CTNNB1 gene during malignant transformation and development of metastasis[10]. Studies indicate that patients with mutations in the Wnt/ $\beta$-catenin pathway, including CTNNB1 mutations, have an inferior response to regorafenib[11] and particularly to immunotherapy[12]. A murine HCC model supports these clinical findings showing resistance to anti-PD-1 therapy in tumors with activation of the Wnt/ $\beta$-catenin pathway[13].

In the present study we aimed to prospectively monitor and evaluate the concordance between the CTNNB1 p.T41A mutation in ctDNA and tumor tissue in patients with HCC. For this purpose, droplet digital PCR (ddPCR) is a sensitive, feasible and affordable method for detecting specific low frequency mutated alleles in a background of abundant non-mutated alleles[14]. We hypothesized that the combined analysis of ctDNA and tumor tissue DNA may provide a more complete picture of the frequency of $C T N N B 1$ mutation. Further, we aimed to evaluate serial testing of ctDNA in a clinical setting and the correlation of the CTNNB1 mutation to clinical outcome.

\section{Patients And Methods}

\section{Ethical approval}

The study was approved by the Central Denmark Region Committee on Biomedical Research Ethics (no. 1-10-72-240-16) and conducted in accordance with the Declaration of Helsinki.

\section{Patients and sample preparation}

The study included 95 patients with the following inclusion criteria; a clinical diagnosis of HCC; plans to initiate a new treatment modality; and age above 18 years and no synchronous malignancy (apart from non-melanoma skin cancer). All patients gave written informed consent. Patients were recruited from November 2016 through October 2018 at the Department of Hepatology and Gastroenterology or the Department of Oncology, Aarhus University Hospital.

Clinical data on disease stage, previous and new treatment(s), treatment response, and mortality were obtained from patient charts.

Blood samples were collected from each patient before commencing a new treatment, after one month and every six months thereafter for up to three years. Samples were collected in $9 \mathrm{~mL}$ EDTA tubes (Becton Dickinson, Plymouth, United Kingdom) and processed within 4 hours. Samples were centrifuged (1800 g at $4^{\circ} \mathrm{C}$ for 10 minutes), and plasma was carefully removed to avoid contamination and stored at $-80^{\circ} \mathrm{C}$. Tumor tissue was collected from 37 of the patients.

\section{Cell-free DNA (cfDNA) and tumor tissue DNA analysis}


Using QIAamp Circulating Nucleic Acid Kit (Qiagen, Hilden, Germany) cfDNA was extracted from 4-5 mL of plasma and eluted in $100 \mu \mathrm{l}$ of elution buffer. DNA extraction from tissue samples was performed with the DNA Mini Kit (Qiagen) on a QIAsymphony.

Doplet digital PCR (ddPCR) analyses were performed with the CTNNB1 p.T41A and wild type (WT) assay from Bio-Rad using the QX200 AutoDG Droplet Digital PCR system (Bio-Rad, Hercules, CA, USA). Samples were run in triplicates. Each run included a nontemplate control, cell-free DNA (cfDNA) from a healthy donor and a mutation-positive control. QuantaSoft analysis software version v.1.7.4.0917 (Bio-Rad) was used in all data analyses.

The limit of detection (LoD) was determined as previously described by analyzing cfDNA from healthy donors[15]. See Supplementary material for a more detailed description of the methods used.

\section{Statistical analysis}

Statistical analyses were performed in STATA version 16.0 (Stata Corporation) and GraphPad Prism version 8.3.0. All tests were two-sided, and $P$ values $<0.05$ were considered statistically significant. Survival analyses were performed using the Cox proportional hazards regression. Correlations between the mutational status and patient characteristics were evaluated by the chi-squared test. Concordance between the mutational status in plasma and tumor tissue was calculated by Cohen's kappa statistics. Data collection was managed using Research Electronic Data Capture (REDCap) hosted at Aarhus University[16, 17].

\section{Results}

\section{Patient characteristics}

Tabel 1 shows the characteristics of the 95 patients in the study. Median follow-up time was 439 days (IQ range 146-724 days) and 69 (71.5\%) patients died within the follow-up period.

Females were more often CTNNB1 mutation positive and there was a trend towards association with Hepatitis $C$ virus infection etiology in plasma mutation positive patients.

\section{Mutational status and clinical course of disease}

A total of 37 ( 8 fresh frozen and 29 formalin-fixed, paraffin-embedded) tissue samples were analyzed and three samples were positive for the CTNNB1 mutation (8.1\%). In the inclusion plasma sample 9 patients out of 95 (9.5\%) were positive for the CTNNB1 p.T41A mutation.

The agreement between plasma and tissue mutational status was $91.7 \%$ (kappa value $0.52, \mathrm{P}=0.0007$ ). Adding results from plasma analyses increased the CTNNB1 mutation detection rate to $13.5 \%(5 / 37)$ in the subgroup of patients with tumor tissue available. 
Two patients were positive in plasma, but negative in tissue. In both cases, the biopsies were from large inhomogeneous tumors $(6.2 \mathrm{~cm}$ and $14.5 \mathrm{~cm}$ respectively). Another patient had a positive tissue sample but negative plasma sample at inclusion. Follow up revealed a negative plasma sample at week 4 and week 26 but at week 104 the mutation was detectable, coinciding with progression of disease.

There was no correlation between CTNNB1 mutation status and mortality (HR 0.62 (0.25-1.54) P=0.30, adjusted for tumor, node, metastasis (TNM) stage, sex and age). Figure 1 shows the changes in concentration of mutated alleles during the course of follow up in relation to treatment and response in the five patients positive for CTNNB1 mutation in plasma at inclusion with follow-up samples available.

The patient depicted in Figure 1A, had two separate intrahepatic tumors on a background of hepatitis $\mathrm{C}$ virus, and had a resection of one tumor and radiofrequency ablation of the other tumor. The resected tumor was positive for the CTNNB1 mutation, while there was no tissue available from the ablated tumor. The patient later had recurrent disease at the ablation site, but without detectable CTNNB1 mutation in plasma on subsequent follow up samples.

\section{Discussion}

In this study we applied ddPCR to detect the CTNNB1 p.T41A hotspot mutation in plasma and tumor tissue from HCC patients at inclusion and during three years of follow-up. We showed that it is feasible to detect the CTNNB1 p.T41A mutation in both plasma and tumor samples using ddPCR, and the serial testing of ctDNA indicated a clonal evolution of HCC or arising multicentric tumors with separate genetic features. We did not detect any difference in mortality depending on CTNNB1 mutational status, however, this study was not designed to evaluate the efficacy of systemic treatment.

At inclusion, we detected the CTNNB1 p.T41A mutation in plasma in $9.5 \%$ of patients. Mutation rate was $13.5 \%$ when combining results from plasma and tumor tissue analysis, leading to a more exhaustive characterization of CTNNB1mutational status with potential implications for future treatment strategy.

Our results are in line with the expected mutation rate, considering previous studies of CTNNB1 p.T41A mutations in HCC patients[18, 9]. CTNNB1 mutations are more frequent in alcohol-related HCC[19] and associated with old age and negatively associated with hepatitis B and elevated alpha-fetoprotein[9], thereby mirroring the etiologic profile of the northern European HCC population.

The majority of previous studies on molecular profiles of individual tumors were performed on early stage tumors and without real-time evaluation of impact on treatment effect. ddPCR is a non-invasive method for CTNNB1 p.T41A mutation that is readily implementable in most larger HCC treatment facilities. ddPCR may supplement next generation sequencing of tumor tissue samples, as it requires a relatively low amount of input ctDNA, results are readily interpretable, and ctDNA reflects the genetic background of the entire tumor burden. Importantly, in this study we detected the CTNNB1 mutation in plasma in two patients in whom biopsy was mutation negative. ddPCR is useful for serial testing of tumor mutational 
status, as demonstrated by one patient with a positive tissue biopsy who became ctDNA positive during follow-up.

Concordance between plasma and tissue mutational status was high. Discrepancy between mutational status in tissue and plasma may be due to tumor heterogeneity, tumor clonal evolution or multicentric tumors. In one case of discrepancy, with CTNNB1 mutation in ctDNA while not present in the index biopsy, we observed progression in satellite tumors during follow-up. The results from the patient in Figure $1 \mathrm{~A}$ could be explained by the presence of multicentric tumors which often occurs in HCC especially on a background of viral hepatitis.

The CTNNB1 p.T41A mutation activates the Wnt pathway and is associated with poor outcome in patients treated with immunotherapy[12]. This underlines the importance of knowing the mutational status in the individual patient. Furthermore, adenomas with mutations in the Wnt/ $\beta$-catenin pathway have higher risk of malignant transformation[20], therefore this noninvasive strategy might also guide decisions regarding treatment or surveillance, although the sensitivity of the assay for small tumors was not evaluated in this study.

The strength of the present study is the number of patients with $\mathrm{HCC}$ and the follow-up of patients during different treatment scenarios. A weakness is the number of tumor tissue available for analysis caused by international guidelines where the HCC diagnosis in cirrhosis patients can be based exclusively on imaging. This study focused on only the most frequent mutation known in CTNNB1 gene. Further studies could apply the multiplex ddPCR method and hereby increase the number of different mutations investigated. Further studies are needed to investigate whether ctDNA can be used to predict and monitor treatment response. This study points at using a combination of tumor mutation profiling in tissue and ctDNA for the most complete characterization. We therefore propose that future studies of HCC therapies should include profiling of ctDNA.

\section{Abbreviations}

cfDNA, cell-free DNA; ctDNA, circulating tumor DNA; ddPCR, droplet digital polymerase chain reaction; FFPE, formalin-fixed paraffin-embedded; HCC, hepatocellular carcinoma; LoD, limit of detection; WT, wildtype.

\section{Declarations}

Acknowledgments: We thank clinical personnel for helping gather samples and laboratory technicians at Department of Hepatology and Gastroenterology, Clinical Biochemistry and Pathology for sample preparation.

Funding: Danish Cancer Society, Aarhus University, Health Research Fund of Central Denmark Region, Einar Willumsen Memorial Fund. 
Conflict of interest: The authors have nothing to disclose in relation to this manuscript.

Ethics approval: The study was approved by the Central Denmark Region Committee on Biomedical Research Ethics (no. 1-10-72-240-16).

Consent to participate: All patients gave written informed consent to participate in the study.

Consent for publication: All patients gave written informed consent to publication of the study results.

Availability of data: The data for the study is available upon request to the corresponding author.

Code availability: Not applicable

Authors contribution: SKO, JK, HG, BW and BSS designed the study. SKO authored the first draft of the manuscript and performed the statistical analysis. MSC and BSS performed the analysis for CTNNB1 mutation and validated the results. SJHD secured data from tissue samples including extraction of DNA and revision of samples. All authors were involved in revising and editing the final manuscript.

\section{References}

1. Wallace MC, Preen D, Jeffrey GP, Adams LA. The evolving epidemiology of hepatocellular carcinoma: a global perspective. Expert review of gastroenterology \& hepatology. 2015;9(6):765-79. doi:10.1586/17474124.2015.1028363.

2. Llovet JM, Ricci S, Mazzaferro V, Hilgard P, Gane E, Blanc JF et al. Sorafenib in advanced hepatocellular carcinoma. The New England journal of medicine. 2008;359(4):378-90. doi:10.1056/NEJMoa0708857.

3. Bruix J, Qin S, Merle P, Granito A, Huang YH, Bodoky G et al. Regorafenib for patients with hepatocellular carcinoma who progressed on sorafenib treatment (RESORCE): a randomised, doubleblind, placebo-controlled, phase 3 trial. Lancet. 2017;389(10064):56-66. doi:10.1016/s01406736(16)32453-9.

4. Finn RS, Qin S, Ikeda M, Galle PR, Ducreux M, Kim TY et al. Atezolizumab plus Bevacizumab in Unresectable Hepatocellular Carcinoma. The New England journal of medicine. 2020;382(20):1894905. doi:10.1056/NEJMoa1915745.

5. Rebouissou S, Nault JC. Advances in molecular classification and precision oncology in hepatocellular carcinoma. J Hepatol. 2020;72(2):215-29. doi:10.1016/j.jhep.2019.08.017.

6. Lin DC, Mayakonda A, Dinh HQ, Huang P, Lin L, Liu X et al. Genomic and Epigenomic Heterogeneity of Hepatocellular Carcinoma. Cancer research. 2017;77(9):2255-65. doi:10.1158/0008-5472.can-162822.

7. Lee JS. The mutational landscape of hepatocellular carcinoma. Clinical and molecular hepatology. 2015;21(3):220-9. doi:10.3350/cmh.2015.21.3.220. 
8. Gao C, Wang Y, Broaddus R, Sun L, Xue F, Zhang W. Exon 3 mutations of CTNNB1 drive tumorigenesis: a review. Oncotarget. 2018;9(4):5492-508. doi:10.18632/oncotarget.23695.

9. Rebouissou S, Franconi A, Calderaro J, Letouze E, Imbeaud S, Pilati C et al. Genotype-phenotype correlation of CTNNB1 mutations reveals different ss-catenin activity associated with liver tumor progression. Hepatology. 2016;64(6):2047-61. doi:10.1002/hep.28638.

10. Vilarinho S, Erson-Omay EZ, Mitchell-Richards K, Cha C, Nelson-Williams C, Harmanci AS et al. Exome analysis of the evolutionary path of hepatocellular adenoma-carcinoma transition, vascular invasion and brain dissemination. J Hepatol. 2017;67(1):186-91. doi:10.1016/j.jhep.2017.03.009.

11. Teufel M, Seidel H, Kochert K, Meinhardt G, Finn RS, Llovet JM et al. Biomarkers Associated With Response to Regorafenib in Patients With Hepatocellular Carcinoma. Gastroenterology. 2019;156(6):1731-41. doi:10.1053/j.gastro.2019.01.261.

12. Harding JJ, Nandakumar S, Armenia J, Khalil DN, Albano M, Ly M et al. Prospective Genotyping of Hepatocellular Carcinoma: Clinical Implications of Next-Generation Sequencing for Matching Patients to Targeted and Immune Therapies. Clinical cancer research : an official journal of the American Association for Cancer Research. 2019;25(7):2116-26. doi:10.1158/1078-0432.ccr-182293.

13. Ruiz de Galarreta M, Bresnahan E, Molina-Sanchez P, Lindblad KE, Maier B, Sia D et al. beta-Catenin Activation Promotes Immune Escape and Resistance to Anti-PD-1 Therapy in Hepatocellular Carcinoma. Cancer discovery. 2019;9(8):1124-41. doi:10.1158/2159-8290.cd-19-0074.

14. Perkins G, Lu H, Garlan F, Taly V. Droplet-Based Digital PCR: Application in Cancer Research. Advances in clinical chemistry. 2017;79:43-91. doi:10.1016/bs.acc.2016.10.001.

15. Milbury CA, Zhong Q, Lin J, Williams M, Olson J, Link DR et al. Determining lower limits of detection of digital PCR assays for cancer-related gene mutations. Biomolecular detection and quantification. 2014;1(1):8-22. doi:10.1016/j.bdq.2014.08.001.

16. Harris PA, Taylor R, Thielke R, Payne J, Gonzalez N, Conde JG. Research electronic data capture (REDCap)-a metadata-driven methodology and workflow process for providing translational research informatics support. Journal of biomedical informatics. 2009;42(2):377-81. doi:10.1016/j.jbi.2008.08.010.

17. Harris PA, Taylor R, Minor BL, Elliott V, Fernandez M, O'Neal L et al. The REDCap consortium: Building an international community of software platform partners. Journal of biomedical informatics. 2019;95:103208. doi:10.1016/j.jbi.2019.103208.

18. Zucman-Rossi J, Villanueva A, Nault JC, Llovet JM. Genetic Landscape and Biomarkers of Hepatocellular Carcinoma. Gastroenterology. 2015;149(5):1226-39.e4. doi:10.1053/j.gastro.2015.05.061.

19. Letouze E, Shinde J, Renault V, Couchy G, Blanc JF, Tubacher E et al. Mutational signatures reveal the dynamic interplay of risk factors and cellular processes during liver tumorigenesis. Nature communications. 2017;8(1):1315. doi:10.1038/s41467-017-01358-x. 
20. Nault JC, Couchy G, Balabaud C, Morcrette G, Caruso S, Blanc JF et al. Molecular Classification of Hepatocellular Adenoma Associates With Risk Factors, Bleeding, and Malignant Transformation. Gastroenterology. 2017;152(4):880-94.e6. doi:10.1053/j.gastro.2016.11.042.

\section{Tables}


Table 1

Patient characteristics

\begin{tabular}{|c|c|c|c|}
\hline & \multicolumn{3}{|l|}{ Plasma } \\
\hline & CTNNB1 mutation positive & CTNNB1 mutation negative & $P$ value \\
\hline All & $(n=9)$ & $(n=86)$ & \\
\hline Age & $68(64-82)$ & $72(66-78)$ & 0.992 \\
\hline \multicolumn{4}{|l|}{ Sex } \\
\hline Female & $5(55.6 \%)$ & $19(22.1 \%)$ & 0.028 \\
\hline Male & $4(44.4 \%)$ & $67(77.9 \%)$ & \\
\hline \multicolumn{4}{|l|}{ Cirrhosis } \\
\hline Yes & $6(66.7 \%)$ & $54(62.8 \%)$ & 0.918 \\
\hline No & $3(33.3 \%)$ & $25(29.1 \%)$ & \\
\hline Unknown & - & $7(8.1 \%)$ & \\
\hline \multicolumn{4}{|l|}{ Child Pugh stage } \\
\hline Non-cirrhosis & $3(33.3 \%)$ & $25(29.1 \%)$ & 0.527 \\
\hline A & $4(44.4 \%)$ & $52(60.5 \%)$ & \\
\hline B & $5(55.6 \%)$ & $28(32.6 \%)$ & \\
\hline C & - & $5(5.8 \%)$ & \\
\hline \multicolumn{4}{|l|}{ Cirrhosis etiology } \\
\hline Alcohol & $2(22.2 \%)$ & $29(33.7 \%)$ & 0.802 \\
\hline Hepatitis C & $3(33.3 \%)$ & $10(11.6 \%)$ & 0.071 \\
\hline Non-alcoholic steatohepatitis & - & $4(4.7 \%)$ & 0.509 \\
\hline Other & - & $7(8.1 \%)$ & 0.374 \\
\hline Unknown/no cirrhosis & $4(44.4 \%)$ & $36(41.9 \%)$ & 0.881 \\
\hline TNM stage & & & 0.914 \\
\hline I & $2(22.2 \%)$ & $29(33.7 \%)$ & \\
\hline II & $2(22.2 \%)$ & $18(20.9 \%)$ & \\
\hline III & $2(22.2 \%)$ & $16(18.6 \%)$ & \\
\hline IV & $3(33.3 \%)$ & $23(26.7 \%)$ & \\
\hline
\end{tabular}




\section{Plasma}

\section{Alpha-fetoprotein}

$\begin{array}{lll}<20 \mathrm{ng} / \mathrm{mL} & 5(55.6 \%) & 36(41.9 \%) \\ \geq 20 \mathrm{ng} / \mathrm{mL} & 4(44.4 \%) & 40(46.5 \%) \\ \text { Not available } & - & 10(11.6 \%)\end{array}$

Figures
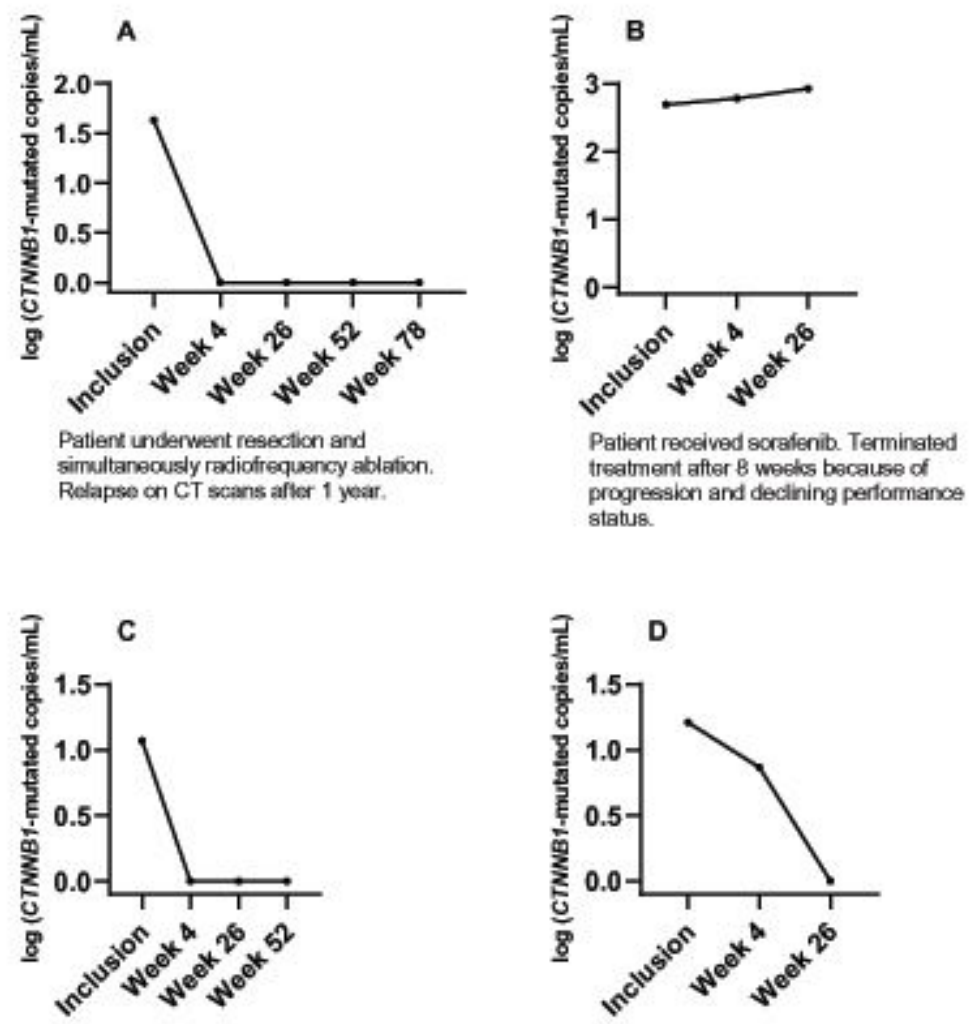

Patient underwent radiofrequency ablation with complate response.

Pafient received Trans Arterial Chemo Embolization with mixed responea.

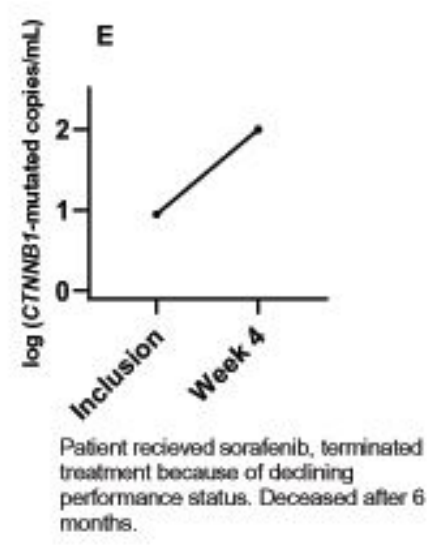




\section{Figure 1}

Correlation between amount of CTNNB1-mutated alleles during follow up and clinical course of disease in five patients.

\section{Supplementary Files}

This is a list of supplementary files associated with this preprint. Click to download.

- Supplementaryfile1.docx 